\title{
The three-loop $\beta$-function of SU(N) lattice gauge theories with overlap fermions
}

\author{
Martha Constantinou * \\ Department of Physics, University of Cyprus, \\ P.O.Box 20537, Nicosia CY-1678, Cyprus \\ E-mail: phpgmc1@ucy.ac.cy
}

\section{Haralambos Panagopoulos}

Department of Physics, University of Cyprus, P.O.Box 20537, Nicosia CY-1678, Cyprus

E-mail: haris@ucy.ac.cy

\begin{abstract}
We briefly report our calculation of the 2-loop coefficient of the coupling constant renormalization function $Z_{g}$ in lattice perturbation theory. The quantity under study is defined through $g_{0}=Z_{g} g$, where $g_{0}(g)$ is the bare (renormalized) coupling constant. The 2-loop expression for $Z_{g}$ can be directly related to the 3-loop bare $\beta$-function $\beta_{L}\left(g_{0}\right)$.

Our calculation is performed using overlap fermions and Wilson gluons, and the background field technique has been chosen for convenience. Our results depend explicitly on the number of fermion flavors $\left(N_{f}\right)$ and colors $(N)$. Since the dependence of $Z_{g}$ on the overlap parameter $\rho$ cannot be extracted analytically, we tabulate our results for different values of $\rho$ in the allowed range $(0<\rho<2)$, focusing on values which are being used most frequently in simulations. Plots of the 1- and 2-loop results for $Z_{g}$ versus $\rho$ exhibit a non-trivial dependence on the overlap parameter.
\end{abstract}

A longer write-up of this work may be found in Ref.[1]:

http://xxx.lanl.gov/abs/0709.4368

The XXV International Symposium on Lattice Field Theory

July 30-4 August 2007

Regensburg, Germany

${ }^{*}$ Speaker. 


\section{INTRODUCTION}

In later years, use of non-ultralocal actions which preserve chiral symmetry on the lattice has become more viable. The two actions which are being used most frequently are overlap fermions based on the Wilson fermion action and domain-wall fermions. Overlap fermions [2] are notoriously difficult to study, both numerically and analytically. Many recent promising results from simulations with overlap fermions have appeared; see, e.g., Refs. [3]-[7]. Regarding analytical computations, the only ones performed thus far have been either up to 1 loop, such as Refs. [8][11], or vacuum diagrams at higher loops [12,13]. The present work is the first one involving non-vacuum diagrams beyond the 1-loop level.

We compute the 2-loop renormalization $Z_{g}$ of the bare lattice coupling constant $g_{0}$ in the presence of overlap fermions. We relate $g_{0}$ to the renormalized coupling constant $g_{\overline{\mathrm{MS}}}$ as defined in the $\overline{\mathrm{MS}}$ scheme at a scale $\bar{\mu}$; at large momenta, these quantities are related as follows

$$
\alpha_{\overline{\mathrm{MS}}}(\bar{\mu})=\alpha_{0}+d_{1}(\bar{\mu} a) \alpha_{0}^{2}+d_{2}(\bar{\mu} a) \alpha_{0}^{3}+\ldots,
$$

( $\alpha_{0}=g_{0}^{2} / 4 \pi, \alpha_{\overline{\mathrm{MS}}}=g_{\overline{\mathrm{MS}}}^{2} / 4 \pi, a$ : lattice spacing). The coefficient $d_{1}$ has been known for a long time; several calculations of $d_{2}$ have also appeared, either in the absence of fermions [14, 15], or using ultra-local fermionic actions $[16,17]$. Knowledge of $d_{2}(\bar{\mu} a)$, along with the 3-loop $\overline{\mathrm{MS}}$ renormalized $\beta$-function allows us to derive the 3-loop bare lattice $\beta$-function, which dictates the dependence of lattice spacing on $g_{0}$. Ongoing efforts to compute the running coupling from the lattice $[18,19]$ have relied on a mixture of perturbative and non-perturbative investigations. As an example, relating $\alpha_{\overline{\mathrm{MS}}}$ to $\alpha_{\mathrm{SF}}$ (SF: Schrödinger Functional scheme), entails an intermediate passage through the bare lattice coupling and the conversion from $\alpha_{\overline{\mathrm{MS}}}$ to $\alpha_{0}$ is carried out perturbatively.

The paper is organized as follows: The theoretical background and the methodology of our perturbative lattice calculations are given in Section II. Section III describes the perturbative expansion of the overlap action. Details on our computation, numerical results and plots of $Z_{g}$ can be found in Section IV.

\section{THEORETICAL BACKGROUND}

The renormalized $\beta$-function describes the dependence of the renormalized coupling constant $g$ on the scale inherent in the renormalization scheme (chosen to be the $\overline{\mathrm{MS}}$ scheme). A bare $\beta$-function is also defined for the lattice regularization $\left(\beta_{L}\left(g_{0}\right)\right)$

$$
\beta\left(g_{\overline{\mathrm{MS}}}\right)=\left.\bar{\mu} \frac{d g_{\overline{\mathrm{MS}}}}{d \bar{\mu}}\right|_{a, g_{0}}, \quad \beta_{L}\left(g_{0}\right)=-\left.a \frac{d g_{0}}{d a}\right|_{g_{\overline{\mathrm{MS}}, \bar{\mu}}}
$$

where $a$ is the lattice spacing, $\bar{\mu}$ the renormalization scale and $g_{\overline{\mathrm{MS}}}\left(g_{0}\right)$ the renormalized (bare) coupling constant. In the asymptotic limit, one can write the expansion of Eq. (2.1) in powers of $g_{0}$

$$
\begin{aligned}
\beta_{L}\left(g_{0}\right) & =-b_{0} g_{0}^{3}-b_{1} g_{0}^{5}-b_{2}^{L} g_{0}^{7}-\ldots \\
\beta\left(g_{\overline{\mathrm{MS}}}\right) & =-b_{0} g_{\overline{\mathrm{MS}}}^{3}-b_{1} g_{\overline{\mathrm{MS}}}^{5}-b_{2} g_{\overline{\mathrm{MS}}}^{7}+\ldots
\end{aligned}
$$

The coefficients $b_{0}, b_{1}$ are well-known universal constants (regularization independent); $b_{i}^{L}(i \geq 2)$ (regularization dependent) must be calculated perturbatively. $\beta_{L}\left(g_{0}\right)$ and $\beta\left(g_{\overline{\mathrm{MS}}}\right)$ can be related using the renormalization function $Z_{g}$, that is 


$$
\beta^{L}\left(g_{0}\right)=\left(1-g_{0}^{2} \frac{\partial \ln Z_{g}^{2}}{\partial g_{0}^{2}}\right)^{-1} Z_{g} \beta\left(g_{0} Z_{g}^{-1}\right)
$$

Computing $Z_{g}^{2}$ to 2 loops

$$
Z_{g}^{2}\left(g_{0}, a \bar{\mu}\right)=1+g_{0}^{2}\left(2 b_{0} \ln (a \bar{\mu})+l_{0}\right)+g_{0}^{4}\left(2 b_{1} \ln (a \bar{\mu})+l_{1}\right)+O\left(g_{0}^{6}\right)
$$

and inserting it in Eq. (2.4), allows us to extract the 3-loop coefficient $b_{2}^{L}$. All quantities in Eq. (2.5), except $l_{1}$ (for overlap fermions), are known. The expansion of Eq. (2.4) in powers of $g_{0}^{2}$ provides the following relation for $b_{2}^{L}$

$$
b_{2}^{L}=b_{2}-b_{1} l_{0}+b_{0} l_{1}
$$

Thus, the evaluation of $b_{2}^{L}$ requires only a 2-loop lattice calculation, of the quantity $l_{1}$.

The most convenient and economical way to proceed with the calculation of $Z_{g}\left(g_{0}, a \bar{\mu}\right)$ is to use the background field technique, in which the following relation is valid

$$
Z_{A}\left(g_{0}, a \bar{\mu}\right) Z_{g}^{2}\left(g_{0}, a \bar{\mu}\right)=1
$$

where $Z_{A}$ is the renormalized function of the background field. In the lattice version of the background field technique, the link variable takes the form

$$
U_{\mu}(x)=e^{i a g_{0} Q_{\mu}(x)} \cdot e^{i a A_{\mu}(x)}
$$

( $Q_{\mu}$ : quantum field, $A_{\mu}$ : background field). In this framework, instead of calculating $Z_{g}$, it suffices to compute $Z_{A}$. For the above calculation, we consider the background field 1PI 2-point function, both in the continuum and on the lattice; for notation and details, the reader can refer to [14]. The gauge parameter $\lambda$ must also be renormalized, in order to compare lattice and continuum results

$$
\lambda=Z_{Q} \lambda_{0}, \quad Z_{Q}=1+g_{0}^{2} z_{Q}^{(1)}+\ldots
$$

$\left(Z_{Q}\right.$ : renormalization function of the quantum field). Finally, $Z_{g}^{2}$ takes the form

$$
Z_{g}^{2}=\left[1+g_{0}^{2}\left(v_{R}^{(1)}-v^{(1)}\right)+g_{0}^{4}\left(v_{R}^{(2)}-v^{(2)}\right)+\lambda_{0} g_{0}^{4} z_{Q}^{(1)} \frac{\partial v_{R}^{(1)}}{\partial \lambda}\right]_{\lambda=\lambda_{0}}
$$

$\left(\lambda_{0}=1\right.$ : bare Feynman gauge). Here, the only unknown quantity is $v^{(2)}$, the 2-loop 1PI lattice Green's function of the background field; its gluonic contribution $\left(N_{f}=0\right)$ is known. One can find the expressions for the remaining, known quantities in Ref. [1].

The fermionic contributions are associated with the diagrams of Fig. $1\left(v^{(1)}\right)$ and Fig. 2 $\left(v^{(2)}\right)$. In the present work, $v^{(2)}$ is perturbatively calculated for the first time using overlap fermions and Wilson gluons. For completeness, we also compute the coefficient $v^{(1)}$ and compare it with previous results. For overlap fermions, the mass counterterm (the filled circle in Fig. 2) equals zero, by virtue of the exact chiral symmetry of the overlap action; consequently, diagrams 19 and 20 both vanish. Diagrams with infrared divergences become convergent only when grouped together $(6+12$, $7+11,8+18,9+17)$.

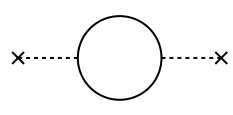

1

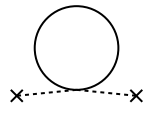

2

Fig. 1: Fermion contributions to the 1-loop function $v^{(1)}$. Dashed lines ending on a cross represent background gluons. Solid lines represent fermions. 


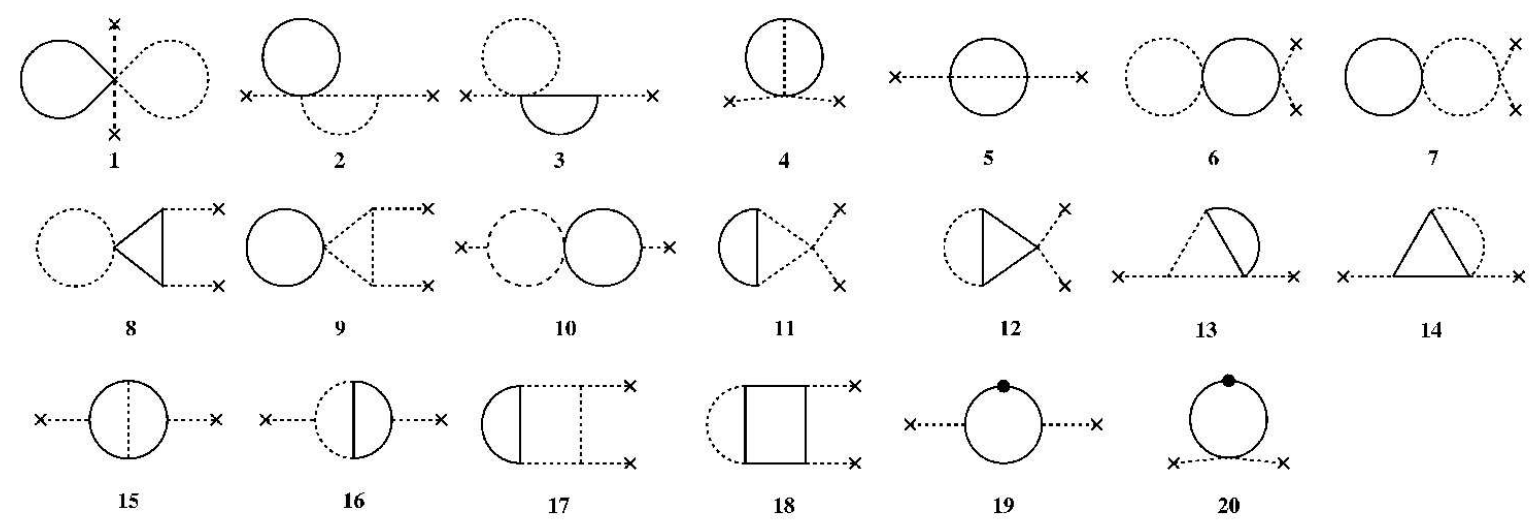

Fig. 2: Fermion contributions to the 2-loop function $v^{(2)}$. Dashed lines represent gluonic fields; those ending on a cross stand for background gluons. Solid lines represent fermions. The filled circle is a 1-loop fermion mass counterterm.

\section{OVERLAP ACTION}

In recent years, overlap fermions are being used ever more extensively in numerical simulations, both in the quenched approximation and beyond. This fact, along with the desirable properties of the overlap action, was our motivation to calculate the $\beta$-function with this type of fermions. The important advantage of the overlap action is that it preserves chiral symmetry while avoiding fermion doubling. It is also $\mathscr{O}(a)$ improved. The main drawback of this action is that it is necessarily non-ultralocal; as a consequence, both numerical simulations and perturbative calculations are extremely difficult and demanding (in terms of human, as well as computer time).

The lattice overlap action is given by

$$
S_{\text {overlap }}=a^{8} \sum_{n, m} \bar{\Psi}(n) D_{N}(n, m) \Psi(m)
$$

where $D_{N}(n, m)$ is the overlap-Dirac operator

$$
D_{N}(n, m)=\rho\left[\frac{\delta_{n, m}}{a^{4}}-\left(X \frac{1}{\sqrt{X^{\dagger} X}}\right)_{n m}\right], \quad X=\frac{1}{a^{4}}\left(D_{W}-\rho\right)
$$

and $D_{W}$ is the Wilson-Dirac operator. The overlap parameter $\rho$ is restricted by the condition $0<$ $\rho<2$ to guarantee the correct pole structure of $D_{N}$. The coupling constant is included in the link variables, present in the definition of $X$, and one must take the perturbative expansion of $X$ in powers of $g_{0}$. This expansion in momentum space takes the form

$$
X\left(p^{\prime}, p\right)=\underbrace{\chi_{0}(p)(2 \pi)^{4} \delta_{P}\left(p^{\prime}-p\right)}_{\text {tree-level }}+\underbrace{X_{1}\left(p^{\prime}, p\right)+X_{2}\left(p^{\prime}, p\right)}_{1-\text { loop }}+\underbrace{X_{3}\left(p^{\prime}, p\right)+X_{4}\left(p^{\prime}, p\right)}_{2-\text { loop }}+O\left(g_{0}^{5}\right)
$$

where $\chi_{0}$ is the inverse fermion propagator and $X_{i}$ are the vertices of the Wilson fermion action ( $p$ $\left(p^{\prime}\right)$ : fermion (antifermion) momentum). Inside of $X_{i}$ 's there appear background and/or quantum gluon fields; their expressions are given in Ref. [1]. Upon substituting the expression for $X_{i}$ 's in the overlap vertices, the latter become extremely lengthy and complicated. For instance, the vertex with Q-Q-A-A- $\Psi-\Psi$ consists of 724,120 terms.

The perturbative expansion of $D_{N}$ leads to the propagator of zero mass fermions and to gluonfermion-antifermion vertices (with up to 4 gluons for the needs of this calculation). After laborious analytical manipulations, the overlap-Dirac operator is expanded into terms with up to 4 gluons as 


$$
D_{N}\left(k_{1}, k_{2}\right)=D_{0}\left(k_{1}\right)(2 \pi)^{4} \delta^{4}\left(k_{1}-k_{2}\right)+\Sigma\left(k_{1}, k_{2}\right)
$$

$D_{0}\left(k_{1}\right)$ is the inverse propagator and $\Sigma\left(k_{1}, k_{2}\right)$ leads to the vertices with up to 4 gluons. Due to the large size of $\Sigma\left(k_{1}, k_{2}\right)$, its explicit form is given in Ref. [1].

\section{RESULTS}

For the algebra involving lattice quantities, we make use of our symbolic manipulation package in Mathematica, with the inclusion of the additional overlap vertices. The first step to evaluate the diagrams is the contraction among vertices; There follow simplifications of the color dependence, Dirac matrices and tensor structures. We use symmetries of the theory, or any other additional symmetry that may appear in particular diagrams, to keep the size of the expression down to a minimum. The external momentum $p$ appears in arguments of trigonometric functions and the extraction of $p$ dependence is divided into two parts: first we isolate terms that give single and double logarithms (a few thousand terms, expressible in terms of known, tabulated integrals) and then for the convergent terms we employ naive Taylor expansion with respect to $p$ up to $\mathscr{O}\left(p^{2}\right)$. The coefficients of terms proportional to $p^{2}$ must be numerically integrated over internal momenta. The integration is performed by optimized Fortran programs which are generated by our Mathematica 'integrator' routine. Each integral is evaluated for several lattices with varying lattice size and for different values of the overlap parameter $\rho$. Finally, we extrapolate the results to infinite size lattices; this procedure introduces an inherent systematic error, which we can estimate quite accurately. Infrared divergent diagrams must be summed up before performing the extrapolation.

We denote the contribution of the $i^{\text {th }}$ 1-loop Feynman diagram to $v^{(1)}(p)$ as $v_{i}^{(1)}(p)$; similarly, contributions of 2-loop diagrams to $v^{(2)}(p)$ are indicated by $v_{i}^{(2)}(p)$

$$
\begin{aligned}
& \widehat{a p}^{2} v_{i}^{(1)}(p)=N_{f}\left[k_{i}^{(0)}+a^{2} p^{2}\left\{k_{i}^{(1)}+k_{i}^{(2)} \frac{\ln a^{2} p^{2}}{(4 \pi)^{2}}\right\}+\mathscr{O}\left((a p)^{4}\right)\right] \\
& \widehat{a p}^{2} v_{i}^{(2)}(p)=N_{f}\left[c_{i}^{(0)}+a^{2} p^{2}\left\{c_{i}^{(1)}+c_{i}^{(2)} \frac{\ln a^{2} p^{2}}{(4 \pi)^{2}}+c_{i}^{(3)}\left(\frac{\ln a^{2} p^{2}}{(4 \pi)^{2}}\right)^{2}+c_{i}^{(4)} \frac{\sum_{\mu} p_{\mu}^{4}}{\left(p^{2}\right)^{2}}\right\}+\mathscr{O}\left((a p)^{4}\right)\right]
\end{aligned}
$$

where $\widehat{p}^{2}=4 \sum_{\mu} \sin ^{2}\left(p_{\mu} / 2\right)$. The index $i$ runs over diagrams, and the coefficients $k_{i}^{(j)}, c_{i}^{(j)}$ depend on the overlap parameter $\rho$. Moreover, $c_{i}^{(j)}=\left[c_{i}^{(j,-1)} / N+c_{i}^{(j, 1)} N\right]$. These coefficients must satisfy conditions coming from comparison with continuum results and usage of Ward Identities; we have checked that these conditions are verified by our results.

$$
\begin{array}{ll}
-\sum_{i} k_{i}^{(0)}=0, \quad \sum_{i} c_{i}^{(0)}=0, \quad \sum_{i} k_{i}^{(2)}=\frac{2}{3}, \quad \sum_{i} c_{i}^{(2)}=\frac{1}{16 \pi^{2}}\left(3 N-\frac{1}{N}\right) \\
-\sum_{i} c_{i}^{(4)}=0, \quad c_{15}^{(3)}=\frac{1}{3 N}, \quad c_{16}^{(3)}=\frac{4}{3} N, \quad c_{17}^{(3)}=-\frac{5}{3} N, \quad c_{18}^{(3)}=\frac{N^{2}-1}{3 N}
\end{array}
$$

In Table I we tabulate the total 1- and 2-loop coefficients $k^{(1)} \equiv \sum_{i} k_{i}^{(1)}, c^{(1,-1)} \equiv \sum_{i} c_{i}^{(1,-1)}$, $c^{(1,1)} \equiv \sum_{i} c_{i}^{(1,1)}$ for several values of the overlap parameter $(0<\rho<2)$, after extrapolating to $L \rightarrow \infty$. The results for $k^{(1)}$ are in good agreement with corresponding results of Ref. [8]. In the Table and all Figures, the errors accompanying our results are entirely due to this extrapolation. In certain 
cases with large systematic errors we extended the integration up to $L=46$. In general, the overlap action leads to coefficients which are very small for most values of $\rho$. As a consequence, systematic errors, which are by and large rather small, tend to be significant fractions of the result for $\rho>1.4$.

\begin{tabular}{|c|l|l|l|}
\hline$\rho$ & \multicolumn{1}{|c|}{$k^{(1)}$} & $c^{(1,-1)}$ & $c^{(1,1)}$ \\
\hline 0.2 & $0.01581702(2)$ & $-0.0044(1)$ & $0.0118(5)$ \\
0.3 & $0.0133504717(2)$ & $-0.00321(6)$ & $0.0045(1)$ \\
0.4 & $0.0116910952(1)$ & $-0.00244(4)$ & $0.0030(1)$ \\
0.5 & $0.0104621922(2)$ & $-0.00191(1)$ & $0.0022(6)$ \\
0.6 & $0.0095058191(2)$ & $-0.001606(6)$ & $0.00176(2)$ \\
0.7 & $0.00874441051(7)$ & $-0.001397(3)$ & $0.00145(1)$ \\
0.8 & $0.00813753230(4)$ & $-0.001241(1)$ & $0.00124(1)$ \\
0.9 & $0.00766516396(3)$ & $-0.001107(1)$ & $0.001051(9)$ \\
1.0 & $0.00732057894(3)$ & $-0.000979(1)$ & $0.000872(3)$ \\
1.1 & $0.00710750173(2)$ & $-0.000849(2)$ & $0.000710(8)$ \\
1.2 & $0.00703970232(7)$ & $-0.000706(3)$ & $0.00052(1)$ \\
1.3 & $0.0071425543(2)$ & $-0.000543(4)$ & $0.00033(3)$ \\
1.4 & $0.0074569183(2)$ & $-0.000335(7)$ & $0.00007(1)$ \\
1.5 & $0.0080467046(1)$ & $-0.00005(1)$ & $-0.0002(1)$ \\
1.6 & $0.0090134204(1)$ & $0.00034(1)$ & $-0.0004(1)$ \\
1.7 & $0.010526080(2)$ & $0.00093(6)$ & $-0.0021(5)$ \\
1.8 & $0.0128914(2)$ & $0.0020(1)$ & $-0.02(3)$ \\
\hline
\end{tabular}

Table 1: Numerical results for the 1-loop $\left(k^{(1)} \equiv \sum_{i} k_{i}^{(1)}\right)$ and 2-loop $\left(c^{(1,-1)} \equiv \sum_{i} c_{i}^{(1,-1)}, c^{(1,1)} \equiv \sum_{i} c_{i}^{(1,1)}\right)$ coefficients for different values of the overlap parameter $\rho$.

We plot the 1-loop coefficient $k^{(1)}$ (Fig. 3) and the 2-loop coefficients $c^{(1,-1)}, c^{(1,1)}$ (Fig. 4) with respect to $\rho$. The extrapolation errors are visible for $\rho \leq 0.4$ and $\rho \geq 1.7$.

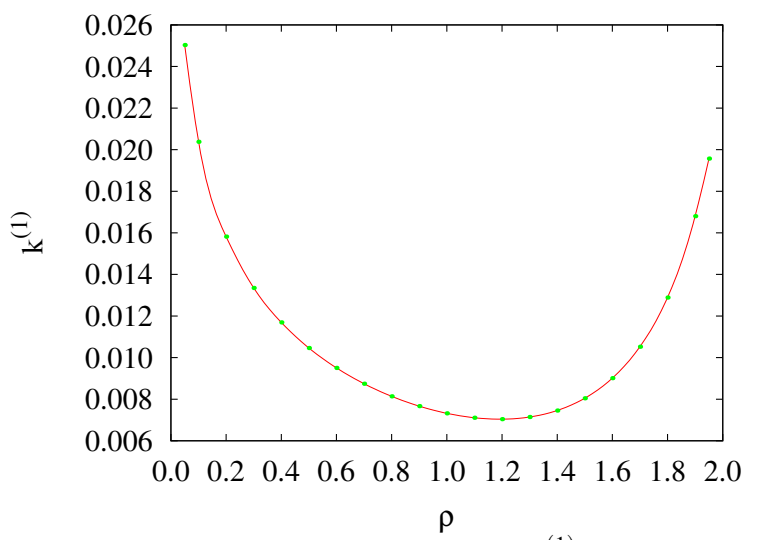

Fig. 3: Plot of the total 1-loop coefficient $k^{(1)}$ for different values of the overlap parameter $\rho$.

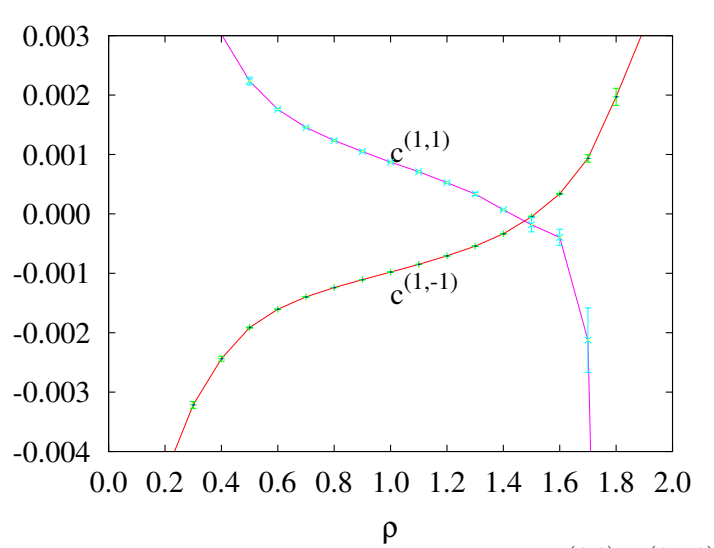

Fig. 4: Plot of the total 2-loop coefficients $c^{(1,1)}, c^{(1,-1)}$ against $\rho$. 
Acknowledgements: This work is supported in part by the Research Promotion Foundation of Cyprus (Proposal Nr: ENI $\Sigma X / 0505 / 45$ ).

\section{References}

[1] M. Constantinou, H. Panagopoulos, QCD with overlap fermions: Running coupling and the 3-loop beta-function, arXiv:0709.4368

[2] R. Narayanan, H. Neuberger, Chiral Fermions on the Lattice,Phys. Rev. Lett. 71 (1993) 3251 [hep-lat/9308011]

[3] E.-M. Ilgenfritz et al., Exploring the structure of the quenched QCD vacuum with overlap fermions, Phys. Rev. D76 (2007) 034506 [arXiv:0705.0018]

[4] J. Bloch et al., An iterative method to compute the sign function of a non-Hermitian matrix and its application to the overlap Dirac operator at nonzero chemical potential, arXiv:0704.3486

[5] JLQCD collaboration: H. Fukaya et al., Two-flavor lattice QCD simulation in the epsilon-regime with exact chiral symmetry, hep-lat/0702003

[6] R. Babich et al., Diquark correlations in baryons on the lattice with overlap quarks, hep-lat/0701023

[7] O. Bär et al., Overlap fermions on a twisted mass sea, hep-lat/0609039

[8] C. Alexandrou, H. Panagopoulos, E. Vicari, Lambda-parameter of lattice QCD with the overlap-Dirac operator, Nucl. Phys. B571 (2000) 257 [hep-lat/9909158]

[9] S. Capitani, Perturbative renormalization for overlap fermions, Nucl. Phys. Proc. Suppl. 106 (2002) 826 [hep-lat/0108028]

[10] T. DeGrand, One loop matching coefficients for a variant overlap action and some of its simpler relatives, Phys. Rev. D67 (2003) 014507 [hep-lat/0210028]

[11] M. Göckeler et al., One-loop Renormalisation of Lattice QCD Operators for Non-forward Matrix Elements: From Clover to Overlap Fermions, PoS ( LAT2006) 161 [hep-lat/0610060]

[12] A. Athenodorou, H. Panagopoulos, The Lattice Free Energy with Overlap Fermions: A Two-Loop Result, Phys. Rev. D70 (2004) 077507 [hep-lat/0408020]

[13] A. Skouroupathis, H. Panagopoulos, Additive and multiplicative renormalization of topological charge with improved gluon/fermion actions, Phys. Rev. D72 (2005) 094509 [hep-lat/0509012]

[14] M. Lüscher and P. Weisz, Computation of the relation between the bare lattice coupling and the MSbar coupling in SU(N) gauge theories, Nucl. Phys. B452 (1995) 234 [hep-lat/9505011]

[15] B. Allés, A. Feo, H. Panagopoulos, The three-loop beta function in $S U(N)$ lattice gauge theories, Nucl. Phys. B491 (1997) 498 [hep-lat/9609025]

[16] C. Christou et al., The three-loop beta function of $S U(N)$ lattice gange theories with Wilson fermions, Nucl. Phys. B525 (1998) 387 [hep-lat/9801007]

[17] A. Bode, H. Panagopoulos, The three-loop beta-fuction of QCD with the clover action, Nucl. Phys. B625 (2002) 198-210 [hep-lat/0110211]

[18] R. Sommer, Non-perturbative QCD: renormalization, $O($ a)-improvement and matching to Heavy Quark Effective Theory Authors:, Lectures given at ILFTN Workshop [hep-lat/0611020]

[19] Q. Mason et al., Accurate Determinations of $\alpha_{s}$ from Realistic Lattice QCD, Phys. Rev. Lett. 95 (2005) 052002 [hep-lat/0503005] 